\title{
ESTANDARIZACIÓN Y VALIDACIÓN DE UNA PRUEBA MOLECULAR RT-LAMP IN HOUSE PARA EL DIAGNÓSTICO DE SARS-COV-2
}

\author{
Oscar Escalante-Maldonado(11,a, Margot Vidal-Anzardo(101,b, Fernando Donaires(101,c, \\ Gilmer Solis-Sanchez (iD2,d, Italo Gallesi(iD),e, Luis Pampa-Espinoza(iD), \\ Maribel Huaringa(101,e , Nancy Rojas-Serrano (D) 1,e , Coralith García (iD),c,

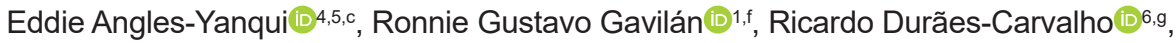 \\ Jairo Mendez-Rico (D) 7,h, César Cabezas (DD1,c , Paulo Vitor Marques-Simas (iD)6,8,i \\ 1 Centro Nacional de Salud Pública, Instituto Nacional de Salud, Lima, Perú. \\ 2 Oficina General de Investigación y Transferencia Tecnológica, Instituto Nacional de Salud, Lima, Perú. \\ 3 Hospital Nacional Cayetano Heredia, Lima, Perú. \\ ${ }^{4}$ Hospital Nacional Arzobispo Loayza, Lima, Perú. \\ 5 Universidad Peruana Cayetano Heredia, Lima, Perú. \\ 6 Universidad de Campinas, Sao Paulo, Brasil. \\ 7 Organización Panamericana de la Salud, Organización Mundial de la Salud, Washington DC, Estados Unidos de América. \\ 8 Universidad Nacional Mayor de San Marcos, Lima, Perú. \\ a Biólogo, doctor en Ciencias Médicas; ${ }^{\mathrm{b}}$ médico cirujano; ${ }^{\mathrm{c}}$ médico cirujano, especialista en Infectología; ${ }^{\mathrm{d}}$ cirujano dentista; \\ e Biólogo; ${ }^{\mathrm{f}}$ biólogo, doctor en Bioquímica y Biología Molecular; $\mathrm{g}$ farmacéutico, doctor en Genética y Biología Molecular; ${ }^{\text {h }}$ doctor en

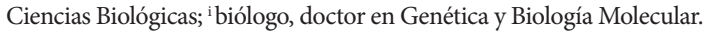

\section{RESUMEN}

Objetivos: Estandarizar una prueba RT-LAMP in house para la detección de SARS-CoV-2 y validarla con muestras de laboratorio y de campo en pacientes con sospecha clínica de COVID-19. Materiales y métodos: Se estandarizó una prueba molecular RT-LAMP in house para la detección de SARS-CoV-2 estableciéndose el límite de detección con células Vero de cepas peruanas aisladas de SARS-CoV-2. Se validó la prueba en laboratorio con 384 muestras de hisopado nasal y faríngeo (HNF) obtenidas entre marzo y julio de 2020. Para la validación de campo se obtuvieron muestras de HNF de 383 casos sintomáticos sospechosos de COVID-19. Todas las muestras fueron evaluadas por RT-LAMP y RT-qPCR. Para la validación de laboratorio y de campo se consideró como estándar de referencia al RT-qPCR, se calcularon medidas de concordancia y rendimiento diagnóstico. Resultados: El límite de detección fue consistente en los casos con umbral de ciclo (Ct) $\mathrm{Ct}<30$ en ambas pruebas, mostrando eficiencia para detectar hasta 1000 copias/ $\mu \mathrm{L}$ del gen diana. Se evidenció robustez con la mitad de las concentraciones de cebadores y $20 \mu \mathrm{L}$ de volumen final. Se identificó ausencia de amplificación para otros coronavirus humanos. La concordancia en laboratorio obtuvo un Kappa de 0,88 (IC 95\%: 0,83-0,93) y en campo fue de 0,89 (IC 95\%: 0,84-0,94); la sensibilidad en laboratorio fue de 87,4\% (IC 95\%: 80,8-92,4) y en campo fue de 88,1\% (IC 95\%: 81,6-92,9), la especificidad en ambos escenarios fue de 98,8\% (IC 95\%: 96,4-99,7). Conclusiones: La prueba RT-LAMP in house fue validada por presentar una adecuada robustez, sin reacciones cruzadas, buena concordancia y rendimiento diagnóstico comparado con el RT-qPCR.

Palabras Clave: COVID-19; Diagnóstico; Técnicas de Diagnóstico Molecular; Reacción en Cadena de la Polimerasa; Estudio de Validación; Sensibilidad y Especificidad; Curva ROC; Valor Predictivo de las Pruebas; Reacciones Cruzadas (fuente: DeCS BIREME).

\section{STANDARDIZATION AND VALIDATION OF AN IN HOUSE RT-LAMP MOLECULAR TEST FOR THE DIAGNOSIS OF SARS-COV-2}

Citar como: Escalante-Maldonado O, Vidal-Anzardo M, Donaires F, Solis-Sanchez G, Gallesi I, PampaEspinoza L, et al. Estandarización y validación de una prueba molecular RT-LAMP in house para el diagnóstico de SARS-CoV-2. Rev Peru Med Exp Salud Publica. 2021;38(1):716. doi: https://doi.org/10.17843/ rpmesp.2021.381.7154.

Correspondencia: Oscar EscalanteMaldonado; Jirón Capac Yupanqui 1400, Jesús María, Perú; oscar.escmal@ gmail.com

Recibido: $20 / 01 / 2021$

Aprobado: $24 / 02 / 2021$

En línea: 09/03/2021

\begin{abstract}
Objectives: To standardize and validate an in-house RT-LAMP test for the detection of SARS-CoV-2, based on laboratory and field assays using samples from COVID-19 suspected patients. Materials and methods: An in-house SARS-CoV-2 RT-LAMP molecular test was standardized, establishing the detection limit with Vero cells of isolated Peruvian strains of SARS-CoV-2, and the robustness to various concentrations of primers. The laboratory validation was performed with 384 nasal and pharyngeal swab samples (UFH) obtained between March and July 2020. The field validation was performed with 383 UFH obtained from COVID-19 suspected symptomatic cases. All samples were tested by RT-LAMP and RT-qPCR. The RT-qPCR was considered as the reference standard test. The concordance measures and diagnostic performance were calculated. Results: The detection limit was consistent in cases with $\mathrm{Ct}<30$ in both tests, showing efficiency to detect up to 1000 copies $/ \mu \mathrm{L}$ of the target gene. Robustness was evidenced with half of the primer concentrations and $20 \mu \mathrm{L}$ of final volume. Absence of amplification was identified for other HCoVs. Concordance showed a kappa index of 0.88 (95\% CI: 0.83-0.93) and 0.89 (95\% CI: 0.84 - 0.94) in laboratory and field settings, respectively. The sensitivity value in the laboratory was $87.4 \%$ (95\% CI: 80.8 - 92.4) and $88.1 \%$ in the field (95\% CI: 81.6 - 92.9). The specificity value in both settings was $98.8 \%$ (95\% CI: 96.4-99.7). Conclusions: The in-house SARS-CoV-2 RT-LAMP test was successfully validated based on its adequate robustness, no cross-reactions, good concordance, and diagnostic performance compared to RT-qPCR.
\end{abstract}

Keywords: COVID-19; Diagnosis; Molecular Diagnostic Techniques; Polymerase Chain Reaction; Validation Study; Sensitivity and Specificity; ROC Curve; Predictive Value of Tests; Cross Reactions (source: MeSH NLM). 


\section{INTRODUCCIÓN}

La COVID-19 es un grave problema de salud pública, alcanzando más de 98 millones de casos confirmados y más de dos millones de muertes en el mundo, al 24 de enero de $2021^{(1)}$. La gran expansión de la enfermedad representa un desafío importante para los países en desarrollo, los cuales deben lidiar con sus brechas sanitarias para el diagnóstico de casos. Estas limitaciones se evidencian en las zonas rurales, donde es necesario desarrollar tecnologías sanitarias descentralizadas para el diagnóstico temprano de casos ${ }^{(2)}$.

La realización de pruebas moleculares requiere considerable inversión financiera y logística, en comparación con otras herramientas de diagnóstico. La prueba de referencia sugerida por la Organización Mundial de la Salud (OMS) para la detección del SARS-CoV-2 es la prueba de reacción en cadena de polimerasa con transcripción inversa en tiempo real (RT-qPCR), la cual requiere de un laboratorio molecular, con infraestructura, equipos y reactivos costosos, así como personal especializado; recursos que no siempre están disponibles en países como el Perú ${ }^{(3,4)}$.

En Perú, al inicio de la pandemia, la prueba de RT-qPCR solo se pudo realizar de forma estandarizada en Lima en el Laboratorio Nacional de Referencia de Virus Respiratorios del Instituto Nacional de Salud (INS). Progresivamente, su procesamiento se extendió a laboratorios regionales de manera descentralizada. Actualmente, existen más de 50 laboratorios a nivel nacional; pero la demanda de estas pruebas, en la práctica, no se ha cubierto plenamente ${ }^{(5)}$.

La imperante necesidad de contar con otras alternativas diagnósticas en fase aguda para la infección por SARS-CoV-2 se ha evidenciado a través del desarrollo de pruebas basadas en el sistema CRISPR/Cas ${ }^{(6)}$, o en el método de amplificación isotérmica mediada por bucle de transcripción inversa (RT-LAMP) ${ }^{(7)}$. Otras pruebas basadas en el método de amplificación isotérmica mediada por bucle (LAMP) se han aplicado en el Perú para enfermedades como zika ${ }^{(8)}$, tuberculosis ${ }^{(9)}$, malaria ${ }^{(10)}$, y dengue ${ }^{(11)}$; evidenciando un adecuado rendimiento. Es más factible y viable su implementación ya que se utilizan equipos más económicos, empleándose de cuatro a seis cebadores, dos / tres hacia adelante $\mathrm{y}$ dos / tres hacia atrás para identificar dianas de ADN, permitiendo sus amplificaciones ${ }^{(12)}$.

La prueba de RT-LAMP se presenta como una alternativa rápida y eficiente para la identificación de casos sospechosos, pues el tiempo de procesamiento de muestras en laboratorio es de aproximadamente 50 minutos, respecto a las cuatro a ocho horas que se requiere para la prueba de RT-qPCR ${ }^{(7)}$.

El presente estudio tiene por objetivo estandarizar en laboratorio una prueba de RT-LAMP desarrollada in house para la detección de SARS-CoV-2, y validarla en campo en

\section{MENSAJES CLAVE}

Motivación para realizar el estudio: La COVID-19 es un grave problema de salud pública en el Perú; es importante desarrollar nuevas metodologías diagnósticas frente al SARS-CoV-2.

Principales hallazgos: La prueba RT-LAMP desarrollada in house demostró un adecuado rendimiento diagnóstico y concordancia respecto a la prueba RT-qPCR, tanto en laboratorio como en campo. No se identificó in silico la existencia de reacciones cruzadas con otros coronavirus. En campo se encontró una reducción del 5,5\% en la sensibilidad, y un aumento de $0,4 \%$ en la especificidad, en sujetos que estaban entre la primera y segunda semana de la enfermedad.

Implicancias: La prueba RT-LAMP in house evaluada es una alternativa eficaz para la detección molecular de SARS-CoV-2.

pacientes con sospecha clínica de COVID-19, obteniendo medidas de rendimiento diagnóstico, tomando como referencia los resultados de la prueba de RT-qPCR.

\section{MATERIALES Y MÉTODOS}

\section{Tipo de estudio}

Se llevó a cabo un estudio transversal que estuvo orientada a evaluar una prueba de RT-LAMP para la detección del SARS-CoV-2 en tres etapas: a) estandarización, b) validación en laboratorio, y c) validación en campo. El estándar de referencia considerado en el estudio fue la RT-qPCR. El diseño de la estandarización fue transversal descriptivo, y el diseño de la validación de laboratorio y de campo fue transversal analítico.

\section{Estandarización}

Esta etapa se llevó a cabo en el Laboratorio Nacional de Referencia de Virus Respiratorio del INS entre junio y julio del 2020. Para evaluar el desempeño de la prueba de RT-LAMP según estándares de la OMS, se usó la cepa de SARS-CoV-2 aislada en células VERO81 a partir de una muestra de hisopado nasofaríngeo (HNF) con diagnóstico molecular por RT-qPCR positivo y muestras de HNF que fueron obtenidas previamente durante vigilancia epidemiológica rutinaria de descarte de la COVID-19. Usando la cepa se evaluó: a) el límite de detección (bajo dilución seriada en base 10), b) la robustez ante cambios en la concentración de cebadores (original y mitad - 0,5P) y reducción porcentual del volumen final de reacción $(20 \%-0,8 \mathrm{~V} ; 40 \%-0,6 \mathrm{~V} ; 50 \%-0.5 \mathrm{~V}$ y $60 \%-0.4 \mathrm{~V})$, y c) la repetibilidad. 


\section{Validación en laboratorio}

El análisis de reacción cruzada fue realizado in silico alineando las secuencias de cebadores externos de la RT-LAMP con secuencias de referencia de los coronavirus humanos conocidos (HCoV) (NC_005831.2, HCoV-NL63; NC_002645.1, HCoV229E; NC_006213.1, HCoV-OC43 cepa ATCC VR-759; NC_006577.2, HCoV-HKU1; NC_004718.3, SARS-CoV-1; NC_019843.3, MERS-CoV coronavirus relacionado con el síndrome respiratorio de Oriente Medio; FJ415324.1, HECoV 4408; NC_045512.2, SARS-CoV-2 de origen chino) ${ }^{(13)}$. Además, los cebadores externos fueron alineados con 194 cepas peruanas disponibles en el GISAID (https://www.gisaid.org/). Todos los experimentos in vitro fueron realizados en triplicado por el mismo operador en las mismas condiciones ambientales y de equipos $^{(13)}$.

Para establecer la validez diagnóstica de la prueba en laboratorio, se calculó un tamaño de muestra mediante la fórmula de estimación del rendimiento diagnóstico por medio del programa Epidat versión 4.2, considerando un valor de $91,489 \%$ de sensibilidad y $99,531 \%$ de especificidad, cifras calculadas según lo reportado por Jiang et al., en el total de su muestra ${ }^{(14)}$; se consideró un nivel de significancia del $95 \%$, error absoluto del $5 \%$ y probabilidad de positividad del 39,5\% (basado en la proporción de resultados positivos obtenidos habitualmente en actividades diagnósticas de los equipos de respuesta rápida de campo por el INS entre el 6 al 8 de julio del 2020 en la jurisdicción de la Dirección de Redes Integradas de Salud (DIRIS) Centro).

Se asumió una tasa de pérdida del $20 \%$ a fin de prever problemas logísticos que pudieran presentarse durante la manipulación y/o análisis de las muestras. Con estos parámetros se estableció la necesidad de contar con, al menos, 379 muestras. Para esta etapa fueron empleadas muestras de HNF, las cuales fueron obtenidas previamente durante evaluaciones epidemiológicas rutinarias de descarte de la COVID-19 entre el inicio de la pandemia y julio de 2020; cuyos viales estaban almacenados en los laboratorios del INS. Todas las muestras evaluadas se encontraban anonimizadas, y correspondían a sujetos con resultados positivos y negativos identificados mediante la RT-qPCR de quienes no se contaba con información adicional.

Las muestras fueron seleccionadas de manera no probabilística por conveniencia, la evaluación por RT-LAMP fue cegada (los evaluadores desconocían el resultado previo por RT-qPCR). El uso de las muestras fue autorizado por resolución jefatural n. ${ }^{\circ} 00006918$, decreto de emergencia sanitaria n. ${ }^{\circ}$ 0064-2020-OGA/INS (7 de abril de 2020) y nota informativa n. ${ }^{\circ}$ 0055-2020 «Plan de Acción del Instituto Nacional de Salud para Prevención, Diagnóstico y Control de COVID-19, en el marco del Decreto Supremo No 0082020-SA».

\section{Extracción de ARN}

La extracción de ARN de todas las muestras se realizó utilizando el kit de purificación de ARN total GenElute ${ }^{\mathrm{mt}}$ (Sigma-Aldrich - Merck), de acuerdo con las instrucciones del fabricante (https://www.sigmaaldrich.com/technical-documents/protocols/biology/viral-rna-purification.html), luego se congeló a $-80^{\circ} \mathrm{C}$ hasta su posterior procesamiento.

\section{Reacción RT-qPCR}

Las reacciones fueron estandarizadas en Rotor-Gene Multiplex RT-PCR Kit (Qiagen, Alemania) usando cebadores y sondas para detección de SARS-CoV-2 $(R d R P)$ y un control interno (GAPDH humano) (canal verde, FAM: 470-510nm y anaranjado, ROX: 585-610nm, respectivamente). Fueron consideradas positivas cuando se obtuvo valores de umbral de ciclo $(\mathrm{Ct})<37$ (FAM) y $\mathrm{Ct}<40$ (ROX), concomitantemente. Las secuencias de cebadores, sondas y condiciones de reacción se encuentran disponibles en el material suplementario. Más información de la prueba RT-qPCR in house disponible en esta dirección: http:// dx.doi.org/10.17504/protocols.io.bsm2nc8e.

\section{Reacción RT-LAMP}

Las reacciones de RT-LAMP se realizaron de acuerdo con lo establecido por Lamb et al. ${ }^{(15)}$, utilizando WarmStart ${ }^{\circ}$ Colorimetric LAMP 2X Master Mix ADN y ARN (Eiken Chemical Co., Ltd., Tokyo, Japan), que contiene un indicador de $\mathrm{pH}$ que permite la visualización colorimétrica. La solidez se probó a partir de la concentración de cebadores estándar y el volumen final de reacción. Fueron usados $44 \mu \mathrm{M}$ de los primers FIP $(16 \mu \mathrm{M})$, BIP $(16 \mu \mathrm{M})$, F3 $(2 \mu \mathrm{M})$, B3 $(2 \mu \mathrm{M})$, LOOP F $(4 \mu \mathrm{M})$, BUCLE B $(4 \mu \mathrm{M})$, y $56 \mu \mathrm{M}$ de agua; además se empleó $20 \mu \mathrm{L}$ de los reactivos MIX-LAMP (12,5 $\mu \mathrm{L})$, MIX-Primers $(2,5 \mu \mathrm{L})$, RNA $(5 \mu \mathrm{L})$, y $5 \mu \mathrm{L}$ de agua. Las reacciones fueron realizadas a $65^{\circ} \mathrm{C}$ por 45 minutos, y a $80^{\circ} \mathrm{C}$ por 5 minutos.

\section{Validación en campo}

Posterior a la validación en laboratorio, se llevó a cabo la evaluación en campo, para lo cual se seleccionaron personas sospechosas de infección por COVID-19 con hasta 15 días de síntomas, quienes acudieron a hospitales de Lima (Hospital Cayetano Heredia, Hospital Hipólito Unanue y Hospital Arzobispo Loayza), y personas que fueron evaluadas por equipos de atención domiciliaria (equipos de respuesta rápida) entre agosto y septiembre de 2020 . Se incluyeron personas mayores de 18 años, con síntomas leves, sin diagnóstico previo de COVID-19 por prueba molecular; se excluyeron a mujeres embarazadas, y a pacientes graves o críticos. El tamaño de muestra para esta etapa se basó en el mismo cálculo realizado para la validación en laboratorio debido a que compartían el mismo objetivo (identificar medidas de rendimiento diagnóstico) aunque en muestras de diferente origen (almacenadas y obtenidas directamente); para la selección de sujetos en campo se siguió un muestreo no probabilístico consecutivo. 
Se registró el sexo, la edad y el cuadro clínico de cada paciente, y el tiempo de enfermedad, en días, desde el inicio de los síntomas. A cada participante se le realizó un HNF, utilizando el kit de muestreo de Yocon Biology Technology Company, que incluye medios de transporte viral e hisopos de dacrón en bandada. Las muestras fueron trasportadas el mismo día al INS mediante contenedores triples con acumuladores de frío, a temperaturas entre $2^{\circ} \mathrm{C}$ a $8^{\circ} \mathrm{C}$. Todas las muestras fueron analizadas siguiendo el procedimiento previamente descrito. Los resultados de la RT-qPCR y RTLAMP se obtuvieron de forma simultánea de dos laboratorios distintos, los evaluadores de cada laboratorio desconocían los resultados de la prueba contraria que analizaban.

\section{Análisis estadístico}

El análisis de los datos se realizó utilizando el paquete estadístico Stata versión 16.1 (Stata Corporation, College Station, Texas, EE. UU.). Se emplearon medidas de resumen de frecuencia y porcentaje para las características clínicas y epidemiológicas de la muestra de sujetos evaluados en la validación de campo. Tanto para la validación de laboratorio como de campo, se determinó el grado de concordancia entre los resultados de la prueba de RT-qPCR y de RT-LAMP mediante el índice Kappa de Cohen. También se calculó la sensibilidad, especificidad, valor predictivo positivo y negativo, valor de la exactitud y el área bajo la curva para la RT-LAMP. Se realizaron análisis estratificados de los resultados de campo según semana de enfermedad ${ }^{(16,17)}$, esto no fue factible para las muestras de laboratorio al no contar con la información sobre el tiempo de enfermedad, los sujetos que no contaron con el tiempo de enfermedad fueron excluidos de la evaluación estratificada. Las medidas fueron calculadas a través de estimadores puntuales e intervalos de confianza al 95\% (IC 95\%), las inferencias se realizaron considerando un nivel de significancia de 0,05.

\section{Consideraciones éticas}

La estandarización y validación en laboratorio no requirió evaluación por el Comité Institucional de Ética en Investigación (CIEI), ya que las muestras empleadas fueron obtenidas en las actividades rutinarias establecidas dentro del plan de acción del INS, resaltando además que las mismas se encontraban anonimizadas. Por su parte, la validación de campo se llevó a cabo mediante un protocolo de investigación aprobado por el CIEI del INS, tal como se muestra en la RD n. ${ }^{\circ}$ 283-2020-OGITT-INS. Todos los sujetos de estudio incluidos en esta fase brindaron su consentimiento informado para participar y se les reportó sus resultados en menos de 72 horas.

\section{RESULTADOS}

\section{Evaluación de desempeño de la RT-LAMP en com- paración con la RT-qPCR}

El límite de detección de SARS-CoV-2 por RT-LAMP fue de 1000 copias $/ \mu \mathrm{L}$; eso indicó que todos los casos en que los valores de $\mathrm{Ct}<30$ fueron concordantes entre RT-qPCR y RT-LAMP (Figura 1, Tabla 1). En las evaluaciones de robustez, se obtuvieron reacciones de alto rendimiento con la

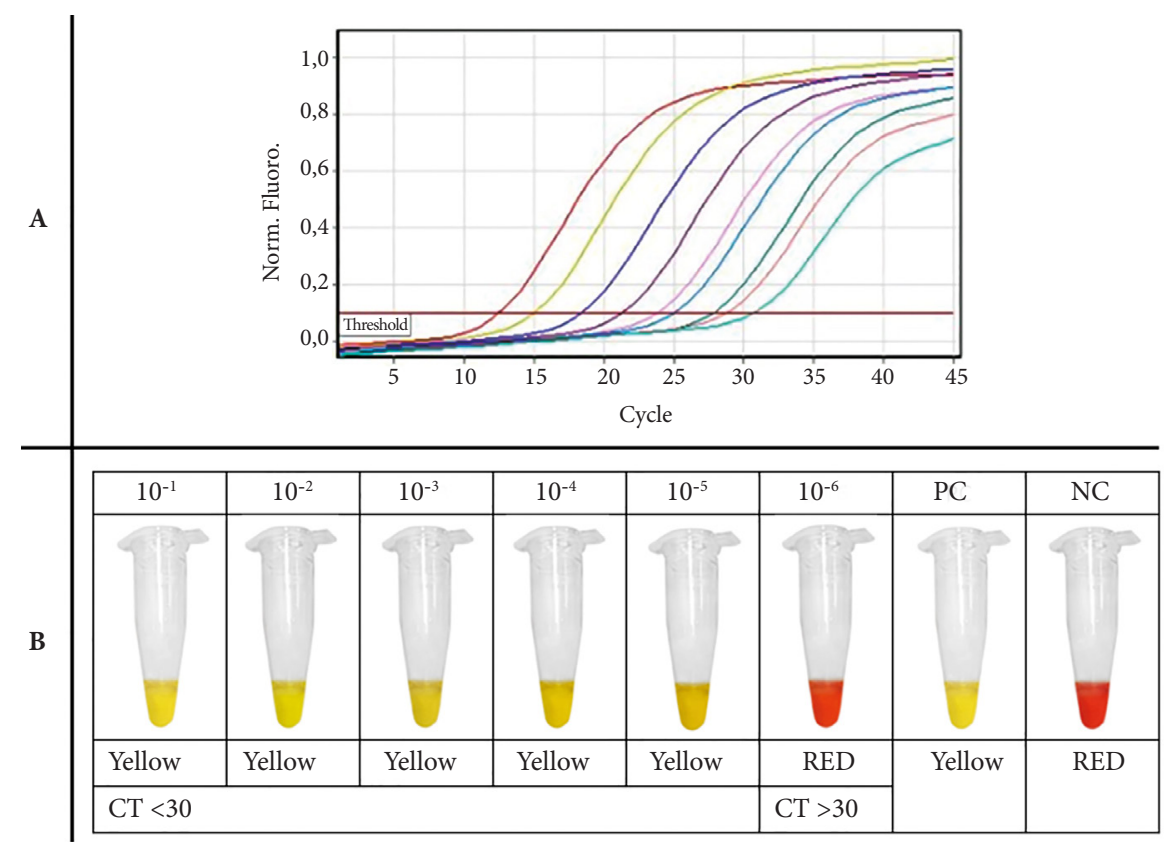

Figura 1. Curva estándar de reacciones de RT-qPCR (panel A) y límite de detección por RT-LAMP (panel B) para la detección de SARS-CoV-2. 
Tabla 1. Comparación del límite de detección entre las reacciones por RT-qPCR y RT-LAMP para detectar la presencia de SARS-CoV-2.

\begin{tabular}{|c|c|c|c|}
\hline Dilución en Serie & $\begin{array}{c}\text { Concentración } \\
(\text { Número de copias/ } \mu \mathrm{L})\end{array}$ & $\begin{array}{l}\text { Valor de Ct } \\
\text { (RT-qPCR) }\end{array}$ & $\begin{array}{l}\text { Cambio de color } \\
\text { (RT-LAMP) }\end{array}$ \\
\hline $10^{-1}$ & $10^{7}$ & 13,6 & Sí \\
\hline $10^{-2}$ & $10^{6}$ & 16,7 & Sí \\
\hline $10^{-3}$ & $10^{5}$ & 20,4 & Sí \\
\hline $10^{-4}$ & $10^{4}$ & 25,0 & Sí \\
\hline $10^{-5}$ & $10^{3}$ & 29,2 & Sí \\
\hline $10^{-6}$ & $10^{2}$ & 35,1 & No \\
\hline $10^{-7}$ & $10^{1}$ & - & No \\
\hline
\end{tabular}

Ct: umbral de ciclo, RT-qPCR: Transcripción Reversa-Reacción en Cadena de la Polimerasa en tiempo real, RT-LAMP: Transcripción reversa-Amplificación Isotérmica Mediada en Lazo

mitad de las concentraciones de cebadores $(0.5 \mathrm{P})$ y con 20 $\mu \mathrm{L}$ de volumen final $(0.8 \mathrm{~V}$ del volumen final de la reacción estándar).

\section{Validación de laboratorio}

El análisis de reacción cruzada realizada in silico, no identificó similitud consistente con las secuencias de referencia de otros coronavirus humanos (NL-63, HKU1, OC43, 229E, SARS-CoV-1, MERS y HECoV, Figura 2). Además, cuando estos mismos cebadores se alinearon con 194 cepas peruanas disponibles en la iniciativa GISAID no hubo exclusión de regiones conservadas, que exhiben una alta similitud y especificidad, lo que puede designarse como ausencia de detección concomitante de otros coronavirus humanos distintos del SARS-CoV-2.

Para la evaluación del rendimiento diagnóstico en laboratorio se incluyeron finalmente 384 muestras, de las cuales $37,2 \%(n=143)$ tenían previamente resultados positivos por RT-qPCR; al obtenerse los resultados por RT-LAMP se identificó una concordancia estadísticamente significativa ( $<<0,001$ ) de 0,88 (IC 95\%: 0,83-0,93) por la prueba de Kappa. Se encontraron tres falsos positivos (FP) con una tasa de falsos positivos (TFP) de 1,2\%; además, se tuvo 18 falsos negativos (FN) con una tasa de falsos negativos (TFN) de 12,6\% (Tabla 2).

La sensibilidad obtenida con las muestras evaluadas fue de $87,4 \%$ (IC 95\%: 80,8-92,4), la especificidad fue de 98,8\% (IC 95\%: 96,4-99,7), el valor predictivo positivo de 97,7\% (IC 95\%: 93,3-99,5), y el negativo de 93,0\% (IC 95\%: 89,1-95,8); el valor de la exactitud de la prueba fue de 94,5\% (IC 95\%: 91,8-96,6), y el área bajo la curva ROC fue de 93,1 \% (IC 95\%: 90,3-95,9) (Tabla 3).

\section{Validación de campo}

Para esta etapa del estudio fueron incluidos 383 sujetos según los aspectos previstos en el protocolo de investigación, de los cuales el 51,7\% ( $\mathrm{n}=198)$ fueron mujeres, el grupo etario más frecuente fue de adultos jóvenes $(n=236,61,6 \%)$. Los síntomas más frecuentes encontrados fueron tos $(n=268$, $70,0 \%)$ y dolor faríngeo $(n=262,68,4 \%)$; la media del tiempo de enfermedad fue de 7,1 (DE: 3,3) días; el 56,1\% se encontraba en la primera semana de enfermedad y el $43,6 \%$ en la segunda semana, se identificó un sujeto $(0,3 \%)$ que no recordaba la fecha de inicio de sus síntomas, el cual fue excluido en la evaluación estratificada por tiempo de enfermedad (Tabla 4).

El 37,3\% ( $n=143)$ de los sujetos evaluados obtuvo resultado positivo mediante RT-qPCR, mientras que la positividad por RT-LAMP fue de 33,7\% ( $n=129)$. La concordancia de los resultados obtenidos entre ambas pruebas tuvo un Kappa de 0,88 (IC 95\%: 0,84-0,94); cuando se estratificó por semana de síntomas se encontró que durante la primera semana este valor fue de 0,92 (IC 95\%: 0,86-0,97), mientras que para la segunda semana se tuvo un Kappa de 0,85 (IC 95\%: 0,76-0,93), en todos los escenarios la concordancia fue estadísticamente significativa $(\mathrm{p}<0,001)$. En la muestra general se encontró $20(5,2 \%)$ casos con resultados discordantes entre ambas pruebas, tres FP con una TFP de 1,3\%, y 17 FN con una TFN de 11,9\%; por su parte en la evaluación por semana de síntomas se tuvo 8 (3,7\%) discordancias en la primera semana y $12(7,2 \%)$ para la segunda, la TFP fue de $1,4 \%$ para la primera semana y $1,0 \%$ para la segunda, mientras que la TFN en la primera semana de síntomas fue de $7,9 \%$ y para la segunda de $16,4 \%$ (Tabla 2 ).

Se encontró que la sensibilidad de la muestra en general fue de $88,1 \%$ (IC 95\%: 81,6-92,9), para el grupo de la primera semana de síntomas este valor fue de $92,1 \%$ (IC $95 \%$ : $83,6-97,0$ ) y el de segunda semana fue de $86,6 \%$ (IC $95 \%$ : 72,5-91,5); en cuanto a la especificidad, se tuvo un valor para la muestra en general de 98,8\% (IC 95\%: 96,4-99,7), para la primera semana de 98,6 (IC 95\%: 94,9-99,8) y para la segunda de 99,0\% (IC 95\%: 94,6-100). El valor predictivo positivo (VPP) y el valor predictivo negativo (VPN) 


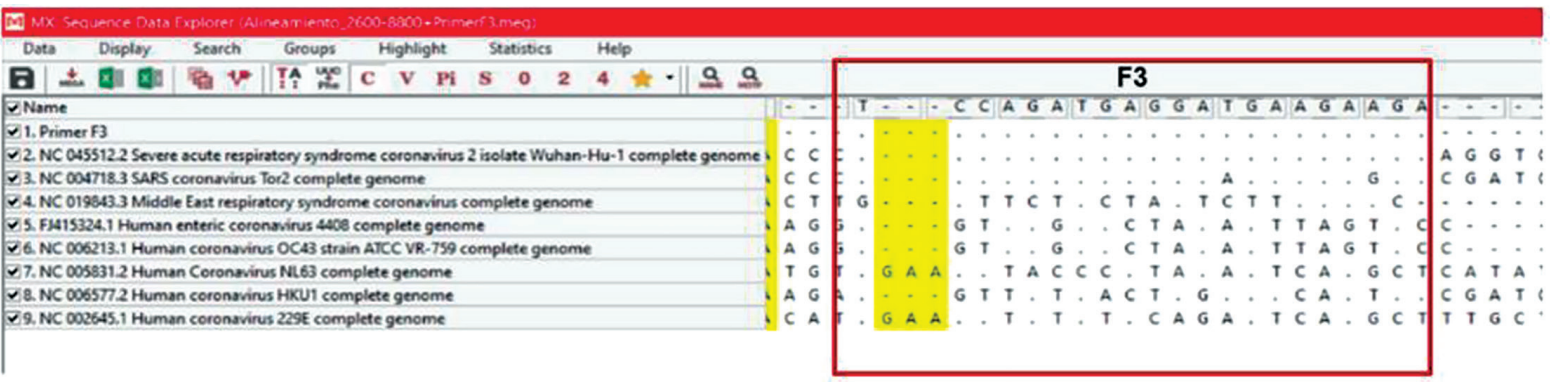

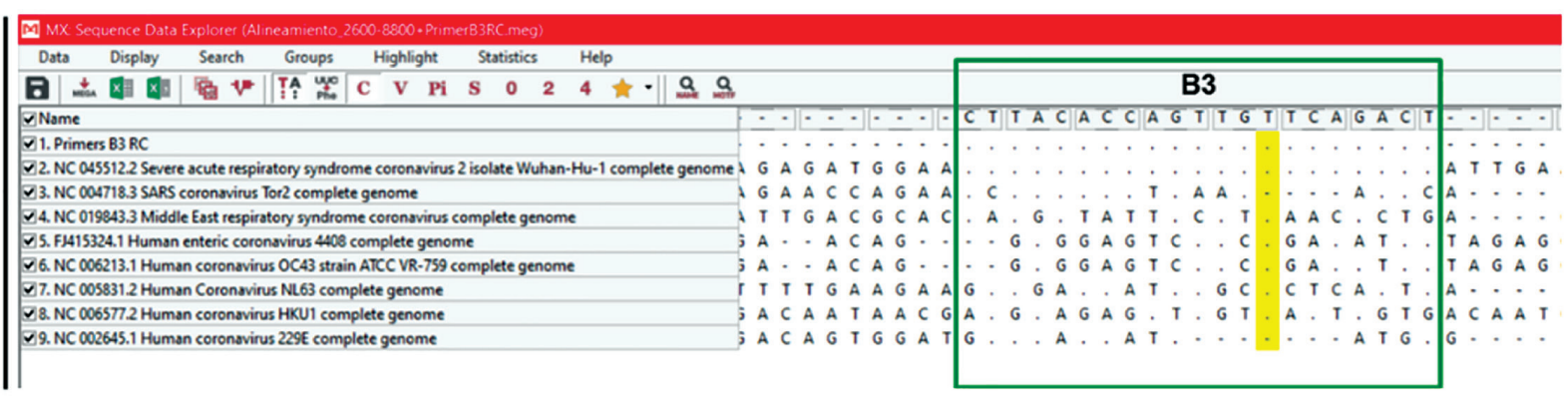
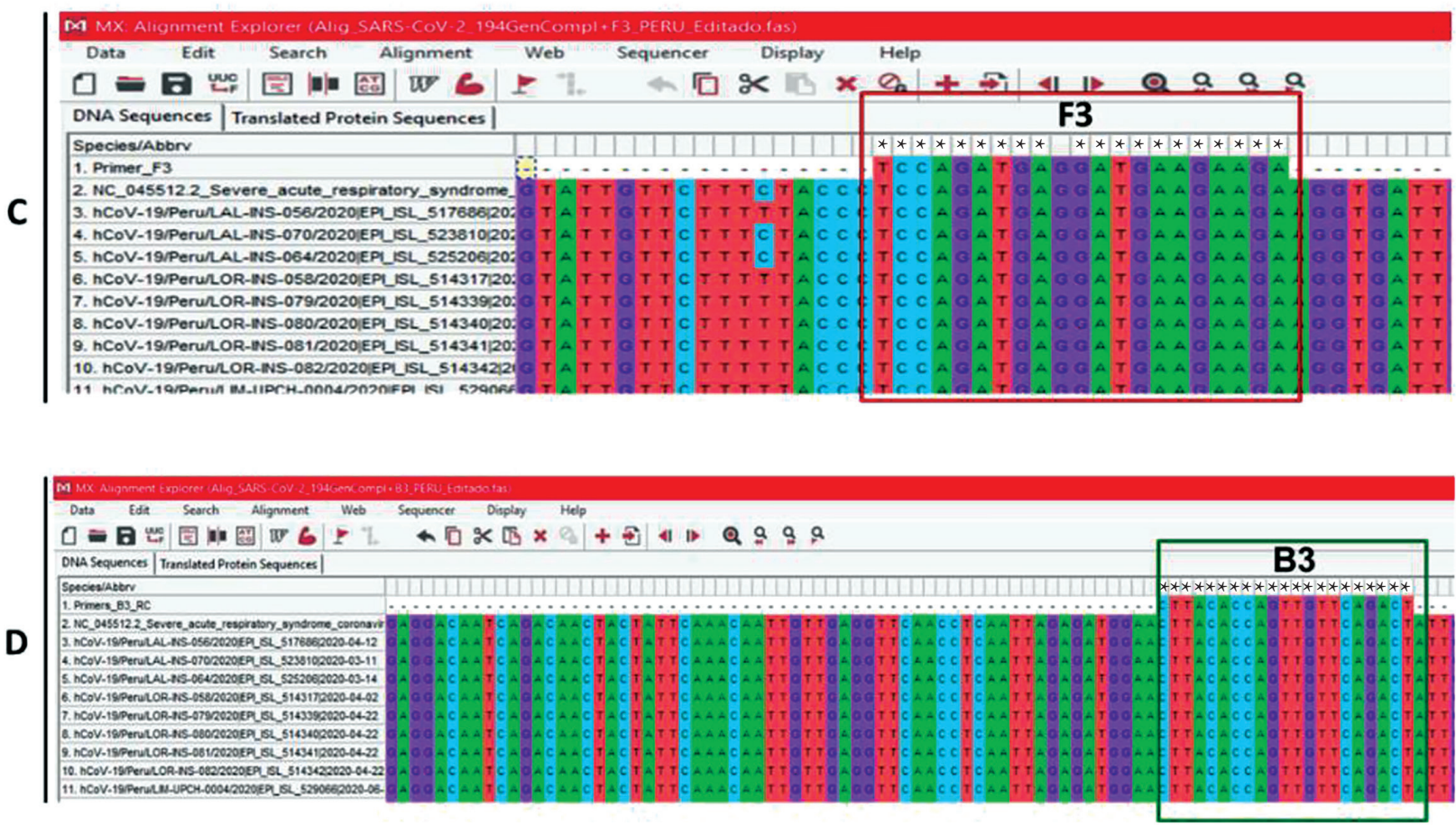

Figura 2: Alineación de múltiples secuencias entre los primers F3 y B3 para RT-LAMP y las secuencias de referencia de todos los coronavirus humanos conocidos, así como con todas las cepas peruanas de SARS-CoV-2 disponibles en la iniciativa GISAID. La alineación fue realizada en ClustalW usando MEGA. La secuencia de primers (panel A - F3, panel B - B3) fueron alineadas con todas las secuencias de referencia de coronavirus humanos conocidos (NC_005831.2, HCoV-NL63; NC_002645.1, HCoV-229E; NC_006213.1, HCoV-OC43 cepa ATCC VR-759; NC_006577.2, HCoV-HKU1; NC_004718.3, SARS-CoV-1; NC_019843.3, MERS; FJ415324.1, HECoV-4408 and NC_045512.2, SARS-CoV-2 Wuhan-Hu-1 aislada) y todas las 194 cepas peruanas de SARS-CoV-2 (panel C - F3, panel D - B3). La columna amarilla en los paneles A y B, y los asteriscos en los paneles C y D, representa regiones conservadoras en el fragmento del gen $n s p 3$ entre todos los coronavirus humanos conocidos y todas las cepas peruanas de SARS-CoV-2 de genoma completo, respectivamente.

para la muestra en general fue de 97,7 (IC 95\%: 93,4- 99,5) y 93,3 (IC 95\%: 89,5-96,1), respectivamente; en la evaluación por semana de síntomas se tuvo que el VPP en la primera semana fue de $97,2 \%$ (IC $95 \%$ : 90,3 - 99,7) y $98,2 \%$
(IC 95\%: 90,6-100) para la segunda, el VPN fue de 95,8\% (IC 95\%: 91,0-98,4) para la primera semana y 90,0 (IC 95\%: $82,8-94,9)$ para la segunda. El área bajo la curva ROC fue de 93,4\% (IC 95\%: 90,7-96,2) en la muestra general, mientras 
Tabla 2. Análisis de concordancia entre los resultados de las evaluaciones en laboratorio y campo obtenidos por las pruebas RT-qPCR y RT-LAMP.

\begin{tabular}{|c|c|c|c|c|c|c|c|c|c|}
\hline \multirow[b]{2}{*}{ RT-LAMP } & \multicolumn{2}{|c|}{ RT-qPCR } & \multirow[b]{2}{*}{ Total } & \multirow[b]{2}{*}{ Kappa (IC 95\%) } & \multirow{2}{*}{$\begin{array}{l}\text { Valor } \\
\text { de p }\end{array}$} & \multicolumn{4}{|c|}{ Resultados } \\
\hline & Positivo & Negativo & & & & $\begin{array}{l}\text { Correctos } \\
(\mathrm{VP}+\mathrm{VN})\end{array}$ & $\begin{array}{c}\text { Incorrectos } \\
(\mathrm{FP}+\mathrm{FN})\end{array}$ & $\begin{array}{c}\text { FP } \\
(\text { PFP })\end{array}$ & $\begin{array}{c}\text { FN } \\
(\mathrm{PFN})\end{array}$ \\
\hline \multicolumn{10}{|c|}{ Evaluación de laboratorio } \\
\hline Positivo & 125 & 3 & 128 & $0,880(0,831-0,930)$ & $<0,001$ & $363(94,5 \%)$ & $21(5,5 \%)$ & $3(1,2 \%)$ & $18(12,6 \%)$ \\
\hline Negativo & 18 & 238 & 256 & & & & & & \\
\hline Total & 143 & 241 & 384 & & & & & & \\
\hline \multicolumn{10}{|c|}{ Evaluación de campo } \\
\hline \multicolumn{10}{|c|}{ General } \\
\hline Positivo & 126 & 3 & 129 & $0,886(0,838-0,935)$ & $<0,001$ & $363(94,8 \%)$ & $20(5,2 \%)$ & $3(1,3 \%)$ & $17(11,9 \%)$ \\
\hline Negativo & 17 & 237 & 254 & & & & & & \\
\hline Total & 143 & 240 & 383 & & & & & & \\
\hline \multicolumn{10}{|c|}{$\begin{array}{l}\text { Primera semana de } \\
\text { síntomas }\end{array}$} \\
\hline Positivo & 70 & 2 & 72 & $0,918(0,862-0,974)$ & $<0,001$ & $207(96,3 \%)$ & $8(3,7 \%)$ & $2(1,4 \%)$ & $6(7,9 \%)$ \\
\hline Negativo & 6 & 137 & 143 & & & & & & \\
\hline Total & 76 & 139 & 215 & & & & & & \\
\hline \multicolumn{10}{|c|}{$\begin{array}{l}\text { Segunda semana de } \\
\text { síntomas }\end{array}$} \\
\hline Positivo & 56 & 1 & 57 & $0,847(0,764-0,930)$ & $<0,001$ & $155(92,8 \%)$ & $12(7,2 \%)$ & $1(1,0 \%)$ & $11(16,4 \%)$ \\
\hline Negativo & 11 & 99 & 110 & & & & & & \\
\hline Total & 67 & 100 & 167 & & & & & & \\
\hline
\end{tabular}

IC 95\%: intervalo de confianza al 95\%, VP: verdadero positivo, VN: verdadero negativo, FP: falso positivo, FN: falso negativo, PFP: proporción de falsos positivos, PFN: proporción de falsos negativos, RT-qPCR: Transcripción Reversa-Reacción en Cadena de la Polimerasa en tiempo real, RT-LAMP: Transcripción Reversa-Amplificación Isotérmica Mediada en Lazo

que para quienes cursaban primera semana de síntomas fue de 95,3\% (IC 95\%: 92,1-98,5), y 91,3\% (IC 95\%: 86,7-95,9) para los de la segunda.

\section{DISCUSIÓN}

La prueba RT-LAMP desarrollada in house demostró un adecuado rendimiento diagnóstico y concordancia respecto a la prueba RT-qPCR, tanto en laboratorio como en campo. Es necesario contar con herramientas diagnósticas con un adecuado rendimiento diagnóstico, alta viabilidad en campo, que requieran menor logística para la obtención de resultados y que sean comparables al RT-qPCR; todo ello a fin de mejorar la cobertura y satisfacer la demanda existente. Teniendo en cuenta todos estos puntos, el RT-LAMP se muestra como una alternativa viable para todos estos requisitos ${ }^{(18)}$.

La prueba RT-LAMP se fundamenta en una reacción colorimétrica rápida por cambio de $\mathrm{pH}$ en la presencia de amplificación específica, y puede proporcionar resultados en menos de dos horas desde de la extracción del ARN. El equipo de investigadores peruanos del INS seleccionó el protocolo descrito por Lamb et al. ${ }^{(15)}$, para comparar su desempeño diagnóstico frente a la prueba RT-qPCR reco- mendada por la OMS, para detectar el gen $R d R p$, descrito por Corman et al ${ }^{(19)}$.

El presente estudio tuvo dos fases en las que se procesaron 767 muestras clínicas. Los resultados indicaron que la prueba de RT-LAMP tiene un rendimiento diagnóstico similar al del RT-qPCR, donde el límite de detección fue de 1000 copias / $\mu \mathrm{L}$, diez veces menor a la RT-qPCR estandarizada e implementada en la rutina de diagnóstico molecular por el INS. Sin embargo, esta diferencia puede estar asociada a la replicación de los coronavirus en general, y sus correspondientes ARN subgenómicos; esta característica puede ocasionar que genes más cercanos de la extremidad 3' tengan más copias durante la replicación, que aquellos más cerca de la extremidad 5' (20). Los cebadores para RT-LAMP se diseñaron para alinearse en la región ORF1a para detectar un fragmento del gen $n s p 3$ del SARS-CoV-2 y los cebadores para RT-qPCR se diseñaron para la región ORF1b para el fragmento del gen $R d R p$.

A partir de estas características de replicación de la familia Coronaviridae, la OMS ha sugerido que el diagnóstico se debe realizar utilizando cebadores para el gen Nucleocapsid (N) o para los genes ORF1ab. Aun así, dado que el ORF1ab representa dos tercios de todo el genoma (secuencia de refe- 
Tabla 3. Medidas de rendimiento diagnóstico del RT-LAMP en validación en laboratorio y en campo, considerando los resultados de RT-qPCR como estándar de referencia.

\begin{tabular}{|c|c|c|c|c|c|c|c|c|}
\hline \multirow{3}{*}{ Parámetro } & \multirow{2}{*}{\multicolumn{2}{|c|}{$\begin{array}{l}\text { Validación en laboratorio } \\
\qquad(\mathrm{n}=384)\end{array}$}} & \multicolumn{6}{|c|}{ Validación en campo } \\
\hline & & & \multicolumn{2}{|c|}{ General $(n=383)$} & \multicolumn{2}{|c|}{$\begin{array}{l}\text { Primera semana de } \\
\text { síntomas }(n=215)\end{array}$} & \multicolumn{2}{|c|}{$\begin{array}{l}\text { Segunda semana de } \\
\text { síntomas }(n=167)\end{array}$} \\
\hline & $\%$ & IC 95\% & $\%$ & IC 95\% & $\%$ & IC 95\% & $\%$ & IC 95\% \\
\hline Sensibilidad & 87,4 & $80,8-92,4$ & 88,1 & $81,6-92,9$ & 92,1 & $83,6-97,0$ & 86,6 & $72,5-91,5$ \\
\hline Especificidad & 98,8 & $96,4-99,7$ & 98,8 & $96,4-99,7$ & 98,6 & $94,9-99,8$ & 99,0 & $94,6-100$ \\
\hline $\begin{array}{l}\text { Valor predictivo } \\
\text { positivo }\end{array}$ & 97,7 & $93,3-99,5$ & 97,7 & $93,4-99,5$ & 97,2 & $90,3-99,7$ & 98,2 & $90,6-100$ \\
\hline $\begin{array}{l}\text { Valor predictivo } \\
\text { Negativo }\end{array}$ & 93,0 & $89,1-95,8$ & 93,3 & $89,5-96,1$ & 95,8 & $91,0-98,4$ & 90,0 & $82,8-94,9$ \\
\hline Exactitud & 94,5 & $91,8-96,6$ & 94,8 & $92,1-96,8$ & 96,3 & $92,8-98,4$ & 92,8 & $87,8-96,2$ \\
\hline Área bajo la curva & 93,1 & $90,3-95,9$ & 93,4 & $90,7-96,2$ & 95,3 & $92,1-98,5$ & 91,3 & $86,7-95,9$ \\
\hline
\end{tabular}

IC 95\%: intervalo de confianza al 95\%, RT-qPCR: Transcripción Reversa-Reacción en Cadena de la Polimerasa en tiempo real, RT-LAMP: Transcripción ReversaAmplificación Isotérmica Mediada en Lazo

rencia NC_045512.2), se debe considerar que los genes ubicados en el genoma $5^{\prime}$ tienen menos copia durante el ciclo de replicación. Por tanto, el gen $n s p 3$ puede tener una menor cantidad de ARN durante la replicación en comparación con la cantidad de ARN para el gen $R d R p$, lo que justificaría la menor sensibilidad de la prueba RT-LAMP. Para superar estas dificultades, diseñamos un nuevo conjunto de cebadores para otras regiones del genoma, especialmente para $R d R p$. para comparar adecuadamente el rendimiento diagnóstico considerando la misma región genómica y los ensayos están en fase final de validación según los parámetros presentados en este trabajo.

También se demostró mediante análisis in silico que el conjunto de cebadores utilizados para RT-LAMP era realmente específico para detectar las cepas peruanas del SARSCoV-2 y no presentaban reacción cruzada con otros coronavirus humanos en prueba molecular. Este punto es una limitación de este estudio porque el análisis debe realizarse in vitro utilizando muestras clínicas, lo que no fue posible debido a que el INS no cuenta con muestras clínicas positivas para otros coronavirus humanos. Debido a la necesidad de evaluar rápidamente el desempeño de este método de diagnóstico y finalmente comenzar a trasferir esta tecnología a los puntos de atención, la alternativa de verificar la ocurrencia de reacción cruzada medida por análisis in silico fue la más apropiada y científicamente factible en el momento.

La identidad perfecta en la región de alineación de cebadores $\mathrm{F} 3$ y $\mathrm{B} 3$ con todas las cepas peruanas de SARS-CoV-2 disponibles también indicó una detección específica y posiblemente ningún resultado falso negativo debido a la especificidad de los cebadores.

La evaluación de la robustez de este protocolo incluyó variables como la concentración de cebadores y el volumen final de reacción. Esta estrategia consideró la posibilidad de que las reacciones fueron realizadas por personas que no tienen contacto rutinario con técnicas de biología molecular. Dado que el rendimiento de las reacciones no se vio comprometido al utilizar la mitad de las concentraciones de cebadores y el $80 \%$ del volumen final de reacción, se pueden cometer errores técnicos durante el pipeteo de pequeño volumen, sin que esto comprometa los resultados.

La RT-LAMP presentó una alta sensibilidad y especificidad tanto en laboratorio como en campo, obteniéndose resultados similares a los reportados por $\mathrm{Hu}$ et al. $(88,57 \%$ y $98,98 \%$, respectivamente) ${ }^{(21)}$, y menor a lo descrito por Jiang et al. (91,4\% y 99,5\%, respectivamente) ${ }^{(14)}$, así como por Kitagawa et al. (100\% y $97,6 \%$, respectivamente) ${ }^{(22)}$. Estas diferencias podrían estar asociadas a los valores de Ct utilizados para establecer la positividad por RT-qPCR; además, solo las muestras positivas que presentaron valores de $\mathrm{Ct}>30$ discreparon de las obtenidas por la RT-LAMP en este estudio.

Los valores de concordancia obtenidos entre ambas pruebas indicaron la aplicabilidad del protocolo de RT-LAMP como una alternativa a la RT-qPCR. La RT-LAMP podría implementarse en el primer nivel de atención, llegando a ser útil para identificar pacientes infectados en la fase de trasmisión activa.

Se encontró que la prueba de RT-LAMP tuvo un VPP de $97,7 \%$, de manera similar a lo reportado por jiang et al. ${ }^{(14)}$, y muy superior a lo mencionado por Hu et al. (VPP: 91,18\%) ${ }^{(21)}$; debemos señalar que este último estudio evaluó 329 muestras de casos asintomáticos, a diferencia de nuestro estudio en el que se tomaron muestras de casos sintomáticos. De igual forma, la prueba de RT-LAMP tuvo un VPN de 93,3\%, lo cual es inferior a lo reportado por Jiang et al. ${ }^{(14)}$, quienes encontraron un VPN del 98,1\%.

El grado de concordancia en la identificación de SARSCoV-2 entre RT-qPCR y RT-LAMP en la valoración clínica fue del $94,8 \%$, resultado similar a lo descrito en otros estudios como el de Lu et al. ${ }^{(23)}$, y Kitagawa et al. ${ }^{(22)}$, donde fueron superiores al 
Tabla 4. Características clínicas y epidemiológicas de los sujetos evaluados en campo.

\begin{tabular}{|c|c|c|}
\hline Características & $\mathbf{n}$ & $\%$ \\
\hline \multicolumn{3}{|l|}{ Sexo } \\
\hline Masculino & 185 & 48,3 \\
\hline Femenino & 198 & 51,7 \\
\hline \multicolumn{3}{|l|}{ Grupo etario (años) } \\
\hline Joven $(\leq 29)$ & 62 & 16,2 \\
\hline Adulto joven (30-59) & 236 & 61,6 \\
\hline Adulto mayor $(\geq 60)$ & 85 & 22,2 \\
\hline \multicolumn{3}{|l|}{ Signos y Síntomas } \\
\hline Ageusia & 19 & 5,0 \\
\hline Anosmia & 37 & 9,7 \\
\hline Cefalea & 214 & 55,9 \\
\hline Congestión nasal & 127 & 33,2 \\
\hline Diarrea & 80 & 20,9 \\
\hline Dificultad respiratoria & 90 & 23,5 \\
\hline Dolor articulaciones & 27 & 7,0 \\
\hline Dolor de garganta & 262 & 68,4 \\
\hline Dolor Muscular & 113 & 29,5 \\
\hline Dolor Pecho & 67 & 17,5 \\
\hline Fiebre/escalofrío & 179 & 46,7 \\
\hline Irritabilidad/confusión & 2 & 0,5 \\
\hline Malestar general & 232 & 60,6 \\
\hline Nauseas/vómitos & 46 & 12,0 \\
\hline Tos & 268 & 70,0 \\
\hline \multicolumn{3}{|l|}{ Tiempo de enfermedad } \\
\hline No especificado a & 1 & 0,3 \\
\hline Primera semana & 215 & 56,1 \\
\hline Segunda semana & 167 & 43,6 \\
\hline \multicolumn{3}{|l|}{ Resultado por RT-PCR } \\
\hline Negativo & 240 & 62,7 \\
\hline Positivo & 143 & 37,3 \\
\hline \multicolumn{3}{|l|}{ Resultado por RT-LAMP } \\
\hline Negativo & 254 & 66,3 \\
\hline Positivo & 129 & 33,7 \\
\hline
\end{tabular}

a Paciente no recuerda el inicio de síntomas.

RT-qPCR: Transcripción Reversa-Reacción en Cadena de la Polimerasa en tiempo real RT-LAMP: Transcripción reversa-Amplificación Isotérmica Mediada en Lazo

90\%. En nuestro estudio encontramos 20 resultados discordantes entre RT-LAMP y RT-qPCR en la valoración clínica, 17 FN y tres FP; Jiang et al. (14), encontraron cinco resultados discordantes, cuatro FN y un FP. Kitagawa et al. ${ }^{(22)}$, reportaron solo dos casos discordantes, que fueron FP. Hu et al. ${ }^{(21)}$, también identificaron cuatro muestras discordantes (teóricamente FP); sin embargo, estos fueron confirmados como positivos para SARS-CoV-2 mediante una prueba de secuenciación genética.
Al evaluar el desempeño de la RT-LAMP por el tiempo de inicio de los síntomas, se encontró que la sensibilidad y el VPN fueron mayores en la primera semana, y aunque el VPP y la especificidad mostraron un incremento hacia la segunda semana, este aumento no fue significativo. Además, se ha informado que RT-qPCR muestra un mayor rendimiento en la primera semana de síntomas; estos hallazgos se pudieron verificar con el área bajo la curva, que de $95,3 \%$ en la primera semana se reduce a $91,3 \%$ a la segunda semana de inicio de los síntomas.

Cabe mencionar que el análisis de datos cuantitativos de reacciones RT-qPCR de muestras del tracto respiratorio inferior es muy confiable, especialmente en casos de pequeñas cantidades de virus ${ }^{(24)}$; sin embargo, este análisis no se pudo realizar en nuestro estudio; identificándose solo un perfil establecido entre los valores de Ct y el momento de inicio de la enfermedad, mostrando que las muestras recolectadas de personas en la primera semana de síntomas presentaron valores de Ct más bajos; esto podría indirectamente ocasionar que la carga viral sea mayor en estas personas.

En laboratorio se tuvo la limitación de no contar con los datos clínicos y epidemiológicos de los sujetos de los cuales provenían las muestras, motivo por el cual no se pudo llevar cabo un análisis estratificado por tiempo de enfermedad. En la evaluación de campo se reconoce el sesgo de memoria de los sujetos incluidos en el estudio, debido a que el dato del tiempo de enfermedad fue obtenido por autorreporte; un único sujeto manifestó no recordar la fecha de inicio de sus síntomas, por lo que fue excluido del análisis estratificado. Los resultados presentaron intervalos de confianza robustos con amplitudes acordes a los estimadores utilizados para la determinación del tamaño de muestra, así como a los valores discordantes obtenidos (FN y FP); estudios en los que se planifiquen tamaños de muestra con menor error absoluto podrían ayudar a aumentar la precisión y por ende reducir los intervalos de confianza.

Finalmente, nuestros datos permiten concluir que la prueba RT-LAMP desarrollado in house ha sido validada como una alternativa conveniente y aceptable para la detección del SARS-CoV-2 en pacientes sintomáticos, estando limitados estos resultados a cuadros clínicos dentro de las dos primeras semanas de enfermedad. Esta prueba se plantea como una alternativa adicional a las ya existentes, lo que ayuda a satisfacer la demanda diagnóstica que se tiene durante la pandemia por la COVID-19. La rápida detección de casos permitiría establecer medidas de control efectivas que se traduzcan en la interrupción de la cadena de contagios y una deseable reducción de la incidencia.

Agradecimientos: Agradecemos a la Organización Panamericana de la Salud(OPS) por proporcionarnoslos reactivosyestablecerla colaboración para realizar las validaciones del experimento. Nuestro reconocimiento a todos los trabajadores del Laboratorio de Microbiología y Biomedicina del INS y a todas las personas de otras instituciones involucradas en la obtención, manipulación y procesamiento de las muestras, en especial 
a Jairo Méndez (OPS), Equipo de Respuesta Rápida (CDC/INS), Lely Solari , Faviola Valdivia, Helen Horna, Gabriel de Lucio, Yanina Zarate, Iris Pompa, Isidro Antipupa, Jhon Mayo, Carina Mantari, Kathia Tarqui, Romeo Pomari, Eduardo Juscamayta, Paquita García, Miryam Palomino, Pamela Rios, Priscila Lope, Johana Balbuena, Víctor Jiménez, Yolanda Angulo, Yuli Barrios, Paul Pachas, Noemi Flores y Ana Zeppilli.

Contribución de los autores: Todos los autores participaron en la concepción y diseño del estudio. LP, CG y EA participaron en la recolección de datos. GS participó en el análisis estadístico de los datos. Todos los autores participaron en la interpretación de los datos, redacción del manuscrito, revisión crítica del manuscrito y aprobaron la versión final. Todos los autores son responsables del

\section{REFERENCIAS BIBLIOGRÁFICAS}

1. World Health Organization. Weekly epidemiological update - 5 January 2021 [Internet]. Ginebra: WHO; 2021 [citado el 25 de enero de 2021]. Disponible en: https://www.who.int/publications/m/item/ weekly-epidemiological-update---5-january-2021.

2. Del Brutto OH, Costa AF, Mera RM, Recalde BY, Bustos JA, García HH SARS-CoV-2-related mortality in a rural Latin American population. Int J Infect Dis. 2020;99:226-8. doi: 10.1016/j.ijid.2020.08.003.

3. Wölfel R, Corman VM, Guggemos W, Seilmaier M, Zange S, Müller MA, et al. Virological assessment of hospitalized patients with COVID-2019. Nature. 2020;581(7809):465-9. doi: 10.1038/s41586-020-2196-x.

4. Organización Mundial de la Salud. Pruebas diagnósticas para el SARSCoV-2: orientaciones provisionales, 11 de septiembre de 2020 [Internet]. Ginebra: OMS; 2021 [citado el 25 de enero de 2021]. Disponible en: https://apps.who.int/iris/handle/10665/335830.

5. Instituto Nacional de Salud. Pruebas moleculares realizadas para el diagnóstico de COVID-19 [Internet]. Lima: INS; 2021 [citado el 25 de enero de 2021]. Disponible en: http://web.ins.gob.pe/es/indicador/ pruebas-moleculares-realizadas-para-el-diagnostico-de-covid-19.

6. Broughton JP, Deng X, Yu G, Fasching CL, Singh J, Streithorst J, et al. Rapid Detection of 2019 Novel Coronavirus SARS-CoV-2 Using a CRISPR-based DETECTR Lateral Flow Assay. MedRxiv Prepr Serv Health Sci. 2020. doi: 10.1101/2020.03.06.20032334.

7. Huang WE, Lim B, Hsu C, Xiong D, Wu W, Yu Y, et al. RT-LAMP for rapid diagnosis of coronavirus SARS-CoV-2. Microb Biotechnol. 2020;13(4):950-61. doi: 10.1111/1751-7915.13586.

8. Escalante-Maldonado O, Gavilán RG, García MP, Marcelo A, Pacheco E, Cabezas $\mathrm{C}$, et al. Desarrollo y validación del método de amplificación isotérmica mediada en lazo para la detección del virus Zika. Rev Peru Med Exp Salud Publica. 2019;36(3):442-7.doi: 10.17843/rpmesp.2019.363.3941.

9. Gray CM, Katamba A, Narang P, Giraldo J, Zamudio C, Joloba M, et al. Feasibility and Operational Performance of Tuberculosis Detection by Loop-Mediated Isothermal Amplification Platform in Decentralized Settings: Results from a Multicenter Study. J Clin Microbiol. 2016;54(8):1984-91. doi:10.1128/JCM.03036-15.

10. Serra-Casas E, Manrique P, Ding XC, Carrasco-Escobar G, Alava F, Gave A, et al. Loop-mediated isothermal DNA amplification for asymptomatic malaria detection in challenging field settings: Technical performance and pilot implementation in the Peruvian Amazon. PloS One. 2017;12(10):e0185742. doi: 10.1371/journal.pone.0185742.

11. Dauner AL, Mitra I, Gilliland T, Seales S, Pal S, Yang S-C, et al. Development of a pan-serotype reverse transcription loop-mediated isothermal amplification assay for the detection of dengue virus. Diagn Microbiol Infect Dis. 2015;83(1):30-6. doi: 10.1016/j.diagmicrobio.2015.05.004.

12. Notomi T, Okayama H, Masubuchi H, Yonekawa T, Watanabe K, Amino $\mathrm{N}$, et al. Loop-mediated isothermal amplification of DNA. Nucleic Acids Res. 2000;28(12):E63. doi: 10.1093/nar/28.12.e63. contenido del artículo.

Conflictos de interés: Todos los autores menos CG, EA, y JM son personal del INS. Todos los autores declaran no tener conflictos de interés en relación con esta publicación. Cesar Cabezas es miembro del comité editor de la RPMESP. RDC cuenta con el apoyo de la Fundación de Investigación de São Paulo (FAPESP), beca n. ${ }^{\circ}$ 2019/01255-9, Brasil.

Financiamiento: Financiado en su totalidad por el INS. El estudio se realizó en el marco de las actividades regulares del INS.

Material suplementario: Disponible en la versión electrónica de la RPMESP.

13. GISAID - Initiative [Internet]. Múnich: GISAID; 2021 [citado el 6 de enero de 2021]. Disponible en: https://www.gisaid.org/.

14. Jiang M, Pan W, Arasthfer A, Fang W, Ling L, Fang H, et al. Development and Validation of a Rapid, Single-Step Reverse Transcriptase Loop-Mediated Isothermal Amplification (RT-LAMP) System Potentially to Be Used for Reliable and High-Throughput Screening of COVID-19. Front Cell Infect Microbiol. 2020;10:331. doi: 10.3389/fcimb.2020.00331.

15. Lamb LE, Bartolone SN, Ward E, Chancellor MB. Rapid detection of novel coronavirus/Severe Acute Respiratory Syndrome Coronavirus 2 (SARS$\mathrm{CoV}-2$ ) by reverse transcription-loop-mediated isothermal amplification. PloS One. 2020;15(6):e0234682. doi: 10.1371/journal.pone.0234682.

16. Carpenter CR, Mudd PA, West CP, Wilber E, Wilber ST. Diagnosing COVID-19 in the Emergency Department: A Scoping Review of Clinical Examinations, Laboratory Tests, Imaging Accuracy, and Biases. Acad Emerg Med OffJ Soc Acad Emerg Med. 2020;27(8):653-70. doi: 10.1111/ acem.14048.

17. Vidal-Anzardo M, Solis G, Solari L, Minaya G, Ayala-Quintanilla B, Astete-Cornejo J, et al. Evaluación en condiciones de campo de una prueba serológica rápida para detección de anticuerpos IgM e IgG contra SARS-CoV-2. Rev Peru Med Exp Salud Pública. 2020;37(2):203-9. doi: 10.17843/rpmesp.2020.372.5534.

18. Kosack CS, Page A-L, Klatser PR. A guide to aid the selection of diagnostic tests. Bull World Health Organ. 2017;95(9):639-45. doi:10.2471/ BLT.16.187468

19. Corman VM, Landt O, Kaiser M, Molenkamp R, Meijer A, Chu DK, et al. Detection of 2019 novel coronavirus (2019-nCoV) by real-time RT-PCR. Eurosurveillance. 2020;25(3). doi: 10.2807/1560-7917. ES.2020.25.3.2000045.

20. Case JB, Bailey AL, Kim AS, Chen RE, Diamond MS. Growth, detection, quantification, and inactivation of SARS-CoV-2. Virology. 2020;548:39-48. doi: 10.1016/j.virol.2020.05.015.

21. Hu X, Deng Q, Li J, Chen J, Wang Z, Zhang X, et al. Development and Clinical Application of a Rapid and Sensitive Loop-Mediated Isothermal Amplification Test for SARS-CoV-2 Infection. mSphere. 2020;5(4):e00808-20. doi: 10.1128/mSphere.00808-20.

22. Kitagawa Y, Orihara Y, Kawamura R, Imai K, Sakai J, Tarumoto $\mathrm{N}$, et al. Evaluation of rapid diagnosis of novel coronavirus disease (COVID-19) using loop-mediated isothermal amplification. J Clin Virol. 2020;129:104446. doi: 10.1016/j.jcv.2020.104446.

23. Lu R, Wu X, Wan Z, Li Y, Jin X, Zhang C. A Novel Reverse Transcription Loop-Mediated Isothermal Amplification Method for Rapid Detection of SARS-CoV-2. Int J Mol Sci. 2020;21(8):2826. doi: 10.3390/ ijms21082826.

24. Yu F, Yan L, Wang N, Yang S, Wang L, Tang Y, et al. Quantitative Detection and Viral Load Analysis of SARS-CoV-2 in Infected Patients. Clin Infect Dis Off Publ Infect Dis Soc Am. 2020; doi: 10.1093/cid/ciaa345. 